\title{
Effect of sterilization by gamma radiation on nano-mechanical properties of teeth
}

Delia S. Brauer ${ }^{\mathrm{a}^{*}}$, Kuniko Saeki ${ }^{\mathrm{a}}$, Joan F. Hilton ${ }^{\mathrm{b}}$, Grayson W. Marshall ${ }^{\mathrm{a}}$, and Sally J. Marshall ${ }^{\mathrm{a}}$

${ }^{a}$ Department of Preventive and Restorative Dental Sciences, UCSF School of Dentistry, Box 0758, San

Francisco CA 94143, USA; 'bepartment of Epidemiology and Biostatistics, UCSF School of Medicine, San Francisco, CA, USA

*corresponding author, present address: Department of Materials, Imperial College London, Exhibition Road, London SW7 2AZ, d.brauer@imperial.ac.uk

\section{SHORT TITLE}

Mechanical properties of teeth after $\gamma$-irradiation

\section{Key words}

Tooth sterilization, gamma radiation; mechanical properties, atomic-force microscopy, nano-indentations, dentin, enamel 


\section{Abstract}

Objectives. Extracted teeth used in dental research need to be considered infective and hence be sterilized without the materials properties being altered. This study examined the effect of gamma radiation on the nano-mechanical properties of dentin and enamel of extracted human third molars.

Methods. Whole teeth were sterilized using gamma radiation doses of $7 \mathrm{kGy}$ and $35 \mathrm{kGy}$, respectively; teeth of the control group were not treated with gamma radiation. Crowns were sectioned occlusally and polished. Elastic modulus and hardness were tested using atomic force microscopy with nano-indentations under wet conditions.

Results. We found no significant dose-response relationship in elastic modulus or hardness in either dentin or enamel.

Significance. Nano-indentation is a common technique for the determination of local mechanical properties in biological hard tissues. Gamma radiation is an efficient way to sterilize extracted teeth while alterations of dentin and enamel mechanical properties are minimized.

\section{Introduction}

Development and testing of restorative dental materials use extracted teeth which are considered to be a potential biological hazard and source of bloodborne pathogens. Thus, infectious agents associated with extracted teeth need to be eliminated. In addition, minimal alterations of structure and properties of the tissue are desirable. In dental research, several sterilization methods are common including autoclaving, chemical heat or dry heat sterilization. However, they might either affect material properties of the teeth or they are unsuitable for amalgam-containing teeth [1].

Gamma radiation is an alternative sterilization method. It is commonly applied for sterilization of medical devices [2;3] and treatment of food [4;5]. In addition, it has successfully been applied for sterilization of bone allografts in orthopedic surgery [6;7]. However, the radiation doses used (25 to $40 \mathrm{kGy}$ ) often reduce the mechanical stability of the bone grafts which might cause the implant to fail [8-10].

Gamma radiation has been shown to sterilize non-carious extracted teeth effectively without affecting the material properties if radiation doses up to $2 \mathrm{kGy}$ are used [11]. If carious teeth are used, higher radiation doses may be necessary and might cause alterations in material structure and properties. Nano-indentation has become a common technique for the determination of local mechanical properties of structural features in biological hard tissues [12-14]. Hence, while investigations of the mechanical properties (hardness, surface morphology and bond strength) on a micro-scale showed no effect of radiation doses up to $25 \mathrm{kGy}$ [15-18], the effect on the mechanical properties on a nano-scale needed to be investigated.

This study examined the effect of gamma radiation on the nano-mechanical material properties of dentin and enamel of extracted non-carious teeth. We hypothesized that sterilization by gamma radiation in doses commonly used for laboratory applications does not affect the nano-mechanical properties of extracted teeth.

\section{Materials and Methods}

\subsection{Tooth sterilization}

Human third molars from subjects requiring extractions as part of dental treatment were used in this study. Teeth were collected following a protocol approved by the UCSF Committee on Human Research. Teeth were stored in Hank's Balanced Salt Solution (HBSS) after extraction. Two groups of teeth were sterilized by use of gamma radiation from a ${ }^{137} \mathrm{Cs}$ source for $40 \mathrm{hr}(7 \mathrm{kGy})$ and $200 \mathrm{hr}(35 \mathrm{kGy})$, respectively. A third group of teeth (the control group) were not treated with gamma radiation. In each group, six teeth were examined. 


\subsection{Sample preparation and nano-indentations}

Crowns were sectioned occlusally using a Buehler Diamond Saw (Buehler, Lake Buff, IL) under running water. The specimens were polished initially with a series of SiC papers and then on polishing cloths with diamond suspensions down to $0.25 \mu \mathrm{m}$. All specimens were stored in HBSS to minimize surface demineralization [12]. Material properties were tested using AFM (atomic force microscopy) with nano-indentations; conducted under wet conditions. The AFM used in this study was a Nanoscope III (Digital Instruments/Veeco, Santa Barbara, CA) with the standard head replaced with a TriboScope indentor system (Hysitron Inc., Minneapolis, MN). A Berkovich Indenter (Hysitron Inc., Minneapolis, MN) was used for both imaging and indentation. Calibration was performed on a standard fused quartz sample with known elastic modulus.

Nano-indentations with a peak load of $400 \mu \mathrm{N}$ on dentin and $2000 \mu \mathrm{N}$ on enamel produced load-deformation curves, from which elastic modulus and hardness were calculated. The hardness, $H$, was calculated on the basis of maximum force divided by the projected contact area at maximum load, while the elastic modulus, $E$, was calculated from the contact stiffness, defined as the slope of the linear portion of the force/displacement curve during unloading near the maximum load. Indentations on intertubular dentin and enamel were performed at two different sites on both buccal and lingual sides with six indentations per site (i.e., 24 indentations per tooth). Thus 18 teeth yielded 432 dentin and 432 enamel measurements of each outcome.

\subsection{Statistical analysis}

Separately for dentin and enamel, the outcomes were examined via graphical methods to identify skewness in the data. No transformations to achieve normality were required. Within tissue type (dentin or enamel), mixed-effects models were fit to determine the dependence of the outcomes on radiation dose $(0,7$, or $35 \mathrm{kGy})$, while accounting for replicate measurements within teeth and sites per tooth. As an aid in identifying outliers, standardized residuals were examined via graphical methods to assess presence of outliers and poor model fit. In the enamel data, five datapoints with standardized residuals $>|3.4|$ were identified and excluded ( 3 were in the buccal location; all were at 35 kGy, Figure 1a). Dentin data were less variable: in models the outcomes as functions of location and dose, only 1 outlier was identified (residual $=3.15$ ). However, in the model regressing elastic modulus on hardness two outliers were found (residuals, 3.4 and 3.7): one buccal at $35 \mathrm{kGy}$ and one lingual at $7 \mathrm{kGy}$ (Figure 1b). In keeping with the threshold used for enamel tissue, these were excluded from the analyses.

Although the purpose of this study was to determine the association between nano-mechanical properties and radiation dose, it was not possible to obtain longitudinal measurements of elastic modulus or hardness within teeth as whole teeth were irradiated but cut into slices and finished for indentations. To reflect periodic cross-sectional measurement of elastic modulus or hardness in distinct teeth, the significance of the association between mechanical properties and radiation dose was assessed via 2-df $F$ statistics allowing for independent measurements across time. As we did not know whether radiation would increase or decrease the mechanical properties, or if such changes would be linear or nonlinear, we used the 2-df F statistic to examine each outcome as function of the dose categories (i.e., a two-sided test that makes no shape assumption). Thus this statistic tests the null hypothesis of no difference among the mean outcomes against the alternative that some difference exists. Results are presented as mean and $95 \%$ confidence interval (CI). 


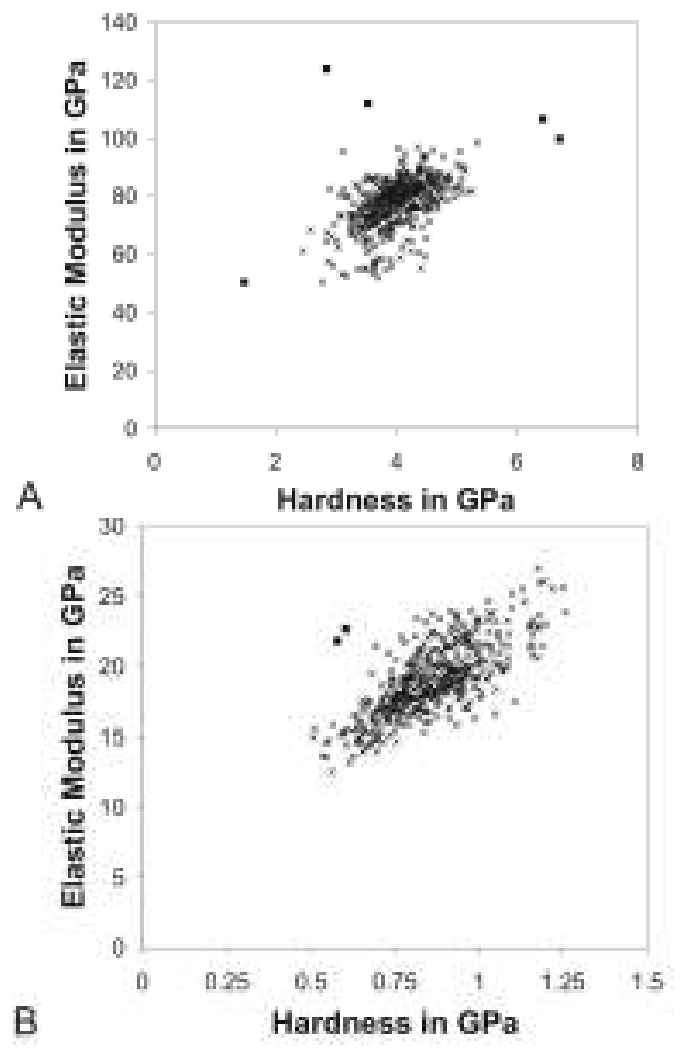

Fig. 1 Elastic modulus vs. hardness for enamel (top) and dentin (bottom) (匹 outliers)

\section{Results}

To determine changes in nano-mechanical properties (elastic modulus and hardness) as a function of radiation dose, we first examined non-irradiated teeth used as baseline data (Table 1). Dentin of non-irradiated teeth showed an elastic modulus of $18.7 \mathrm{GPa}(\mathrm{CI}: 17.5-20.0 \mathrm{GPa})$ and hardness of $0.86 \mathrm{GPa}(\mathrm{CI}: 0.8-0.93 \mathrm{GPa})$. Elastic modulus of enamel was 77.2 GPa (CI: 72.2 - 82.2 GPa), hardness was 4.1 GPa (CI: 3.8 - 4.4 GPa).

Results of dose-response analysis for enamel (Table 1, Figure 2a) showed no statistically significant change with dose according to either elastic modulus or hardness: Hardness results showed a statistically nonsignificant $(p=0.55)$ decrease from $4.1 \mathrm{GPa}(\mathrm{CI}: 3.8-4.4 \mathrm{GPa})$ at $0 \mathrm{kGy}$ to $4.0 \mathrm{GPa}(\mathrm{CI}: 3.7-4.3 \mathrm{GPa})$ at $7 \mathrm{kGy}$ to $3.9 \mathrm{GPa}(\mathrm{CI}: 3.7-4.2 \mathrm{GPa})$ at $35 \mathrm{kGy}$. Elastic modulus of enamel also showed no trend $(p=0.85)$.

Table 1. Effects of radiation dose: results of trend analysis (mean and $95 \%$ confidence interval)

\begin{tabular}{cccc} 
Tissue & $\begin{array}{c}\text { Dose } \\
k G y\end{array}$ & $\begin{array}{c}\text { Elastic Modulus } \\
\text { GPa }\end{array}$ & $\begin{array}{c}\text { Hardness } \\
\text { GPa }\end{array}$ \\
\hline & 0 & $18.7(17.5-20.0)$ & $0.86(0.80-0.93)$ \\
Dentin & 7 & $18.7(17.4-20.0)$ & $0.85(0.79-0.92)$ \\
$(\mathrm{N}=430)$ & 35 & $19.6(18.3-20.9)$ & $0.87(0.80-0.93)$ \\
& & $p=0.51$ & $p=0.93$ \\
\hline Enamel & 0 & $77.2(72.2-82.2)$ & $4.1(3.8-4.4)$ \\
$(\mathrm{N}=427)$ & 7 & $78.9(73.9-83.9)$ & $4.0(3.7-4.3)$ \\
& 35 & $77.3(72.3-82.3)$ & $3.9(3.7-4.2)$ \\
& & $p=0.85$ & $p=0.55$
\end{tabular}


Similarly, in dentin (Table 1, Figure 2b) only a statistically nonsignificant increase with radiation dose was observed for elastic modulus $(p=0.51)$ : Values changed from $18.7 \mathrm{GPa}$ at both $0 \mathrm{kGy}(\mathrm{CI}: 17.5-20.0 \mathrm{GPa})$ and $7 \mathrm{kGy}(\mathrm{CI}$ : $17.4-$ $20.0 \mathrm{GPa}$ ) to $19.6 \mathrm{GPa}$ at $35 \mathrm{kGy}(\mathrm{CI}: 18.3-20.9 \mathrm{GPa})$. Hardness values also showed now statistically significant trend $(p=0.93)$.
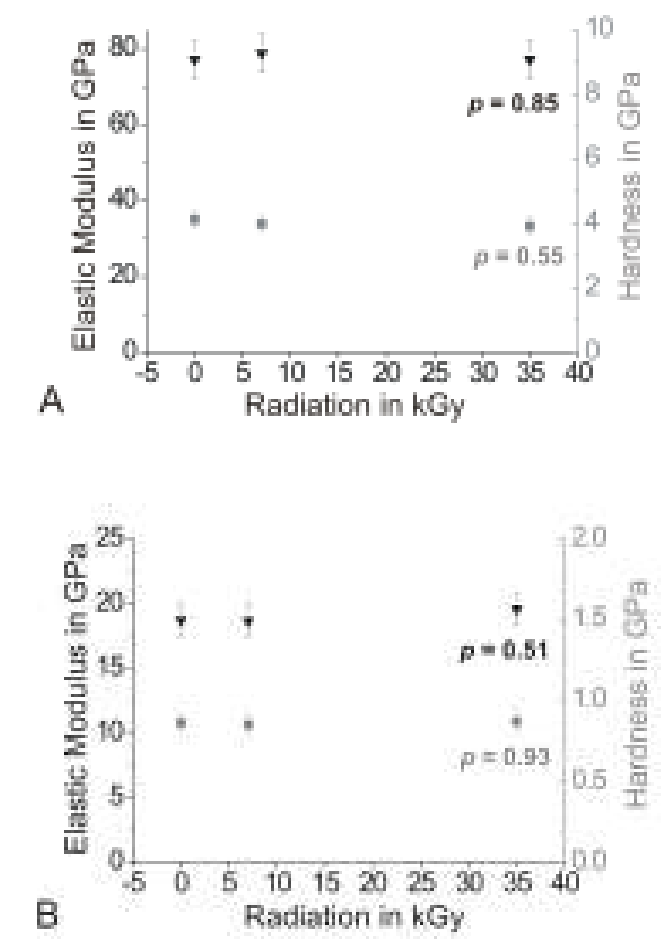

Fig. 2 Elastic modulus ( $\boldsymbol{\nabla}$ ) and hardness ( $\mathbf{\square}$, gray) vs. radiation dose for enamel (top) and dentin (bottom) (error bars: $95 \%$ confidence intervals)

\section{Discussion}

Gamma radiation can cause partial rupture of chemical bonds and reduction of specific ions. For inorganic materials, discoloration or fluorescence caused by introduction of defects caused by gamma radiation are well known [19;20]. While optical properties are affected by low radiation doses, the mechanical properties of inorganic materials are less easily affected. However, gamma radiation has been known to break chemical bonds of polymers including the peptide bonds of proteins. In synthetic polymers, gamma radiation can initiate oxidative aging by stripping of hydrogen from molecules and leaving free radicals [21]. Collagen consists of macromolecular chains of various kinds of amino acids. Its supercoiled triple helix conformation is sensitive to different degrees of radiation. Hence it has been suggested that it may be difficult to sterilize while maintaining the native structural integrity of the collagen fibers [22;23].

As enamel contains considerably less organic material than dentin we assumed that the mechanical properties of enamel would be less easily affected by gamma radiation than those of dentin. However, for the radiation doses used in this investigation, there was no effect on the nano-mechanical properties of dentin or enamel. The influence of the radiation dose on the nano-mechanical properties was investigated in trend analyses without making assumptions about the direction of the effects of radiation or the pattern of the association (e.g., linear or nonlinear), thereby allowing the data to tell us what patterns exist, if any. Results for both elastic modulus and hardness of dentin and enamel (Table 1, Figure 2) showed no 
changes with increasing dose $(\mathrm{p}>0.05)$, even though the highest dose we examined is well above doses previously studied (35 kGy versus $25 \mathrm{kGy}$ ).

Enamel is the hardest and most highly mineralized substance of the body. $96 \%$ of enamel consists of mineral, with water and organic material composing the rest [24]. Dentin is made up of $70 \%$ inorganic materials, $20 \%$ organic materials, and $10 \%$ water by weight; $90 \%$ of the organic material is collagen type 1 and the remaining $10 \%$ ground substance which includes dentine-specific proteins. One possible explanation why the nano-mechanical properties of dentin were not affected by gamma radiation treatment is that while gamma radiation can break chemical bonds, it can also crosslink polymers. Hence breakage in peptide bonds of collagen may be compensated by the crosslinks formed [22]. Another possible explanation is that dentin collagen is reinforced by intrafibrillar mineral and each fibril is surrounded by extrafibrillar mineral. The intrafibrillar mineral, which stiffens the collagen fibrils, dominates the elastic behavior under normal loading conditions [25].

\section{Conclusion}

No significant association between nano-mechanical properties and gamma-radiation dose was found for either dentin or enamel of human third molars. Gamma radiation is a useful way to sterilize extracted teeth while alterations of the mechanical properties are minimized.

\section{Acknowledgements}

Support by NIH/NIDCR Grant P01DE09859 is gratefully acknowledged.

\section{References}

[1] De Wald JP: The use of extracted teeth for in vitro bonding studies: A review of infection control considerations. Dental Materials 1997; 13(2):74-81.

[2] Ley FJ: Radiation Sterilization of Medical Products. International Journal of Nuclear Medicine \& Biology 1982; 9(2):165-166.

[3] Dorpema JW: Review and state-of-the-art on radiation sterilization of medical devices. Radiation Physics and Chemistry 1990; 35(1-3):357-360.

[4] Crawford LM, Ruff EH: A review of the safety of cold pasteurization through irradiation. Food Control 1996; 7(2):87-97.

[5] Josephson ES: Food irradiation and sterilization. Radiation Physics and Chemistry 1981; 18(1-2):223-239.

[6] Vastel L, Meunier A, Siney H, Sedel L, Courpied JP: Effect of different sterilization processing methods on the mechanical properties of human cancellous bone allografts. Biomaterials 2004; 25(11):2105-2110.

[7] Grieb TA, Forng RY, Stafford RE, Lin J, Almeida J, Bogdansky S, Ronholdt C, Drohan WN, Burgess WH: Effective use of optimized, high-dose (50 kGy) gamma irradiation for pathogen inactivation of human bone allografts. Biomaterials 2005; 26(14):2033-2042.

[8] Akkus O, Rimnac CM: Fracture resistance of gamma radiation sterilized cortical bone allografts. Journal of Orthopaedic Research 2001; 19(5):927-934.

[9] Akkus O, Belaney RM: Sterilization by gamma radiation impairs the tensile fatigue life of cortical bone by two orders of magnitude. Journal of Orthopaedic Research 2005; 23(5):1054-1058.

[10] Cornu O, Banse X, Docquier PL, Luyckx S, Delloye C: Effect of freeze-drying and gamma irradiation on the mechanical properties of human cancellous bone. Journal of Orthopaedic Research 2000; 18(3):426-431. 
[11] White JM, Goodis HE, Marshall SJ, Marshall GW: Sterilization of teeth by gamma-radiation. Journal of Dental Research 1994; 73(9):1560-1567.

[12] Habelitz S, Marshall GW, Balooch M, Marshall SJ: Nanoindentation and storage of teeth. Journal of Biomechanics 2002; 35(7):995-998.

[13] Kinney JH, Balooch M, Marshall SJ, Marshall GW, Weihs TP: Hardness and Young's modulus of human peritubular and intertubular dentine. Archives of Oral Biology 1996; 41(1):9-13.

[14] Marshall GW, Balooch M, Gallagher RR, Gansky SA, Marshall SJ: Mechanical properties of the dentinoenamel junction: AFM studies of nanohardness, elastic modulus, and fracture. Journal of Biomedical Materials Research 2001; 54(1):87-95.

[15] Moscovich H, Creugers NHJ, Jansen JA, Wolke JGC: In vitro dentine hardness following gamma-irradiation and freezing. Journal of Dentistry 1999; 27(7):503-507.

[16] Sperandio M, Souza JB, Oliveira DT: Effect of gamma radiation on dentin bond strength and morphology. Brazilian Dental Journal 2001; 12(3):205-208.

[17] Dietrich A, Heimann RB, Willmann G: The colour of medical-grade zirconia (Y-TZP). Journal of Materials Science-Materials in Medicine 1996; 7(9):559-565.

[18] Rodrigues LKA, Cury JA, dos Santos MN: The effect of gamma radiation on enamel hardness and its resistance to demineralization in vitro. Journal of Oral Science 2004; 46(4):215-220.

[19] Ehrt D, Vogel W: Radiation effects in glasses. Nuclear Instruments \& Methods in Physics Research Section BBeam Interactions with Materials and Atoms 1992; 65(1-4):1-8.

[20] Willmann G: The color of bioceramics. Key Engineering Materials 2003; 240-242:785-788.

[21] Blanchet TA, Burroughs BR: Numerical oxidation model for gamma radiation-sterilized UHMWPE: Consideration of dose-depth profile. Journal of Biomedical Materials Research 2001; 58(6):684-693.

[22] Cheung DT, Perelman N, Tong D, Nimni ME: The effect of gamma irradiation on collagen molecules, isolated alpha-chains, and cross-linked native fibers. Journal of Biomedical Materials Research 1990; 24(5):581-589.

[23] Tyan YC, Liao JD, Lin SP, Chen CC: The study of the sterilization effect of gamma ray irradiation of immobilized collagen polypropylene nonwoven fabric surfaces. Journal of Biomedical Materials Research Part A 2003; 67A(3):1033-1043.

[24] Ten Cate AR: Oral Histology: Development, Structure and Function. ed $5^{\text {th }}$, St. Louis, Mosby, 1998.

[25] Kinney JH, Habelitz S, Marshall SJ, Marshall GW: The importance of intrafibrillar mineralization of collagen on the mechanical properties of dentin. Journal of Dental Research 2003; 82(12):957-961. 